\title{
A parameter sensitivity methodology in the context of HIV delay equation models
}

Received: 28 August 2002 / Revised version: 24 January 2004 /

Published online: 20 December 2004 - (c) Springer-Verlag 2004

Abstract. A sensitivity methodology for nonlinear delay systems arising in one class of cellular HIV infection models is presented. Theoretical foundations for a typical sensitivity investigation and illustrative computations are given.

\section{Introduction}

\subsection{Background}

Over the past several years, the use of mathematical models as an aid in understanding features of HIV and other virus infection dynamics has been substantial. Several papers published in the mid nineties provided strong evidence for the high rate of HIV-1 replication and clearance in infected individuals $[22,42,54]$. By the end of the decade, the general consensus was that in vivo, on the order of $10^{10}$ virions are assembled and cleared every day $[31,44]$. In many of these papers, the viral clearance rate $c$ was identified by modeling the disease pathogenesis with a system of deterministic differential equations, numerically calculating a solution, and then fitting the results with experimental data (using a nonlinear least squares (NLS) approach), e.g., see $[42,44]$. The existence of such a high replication/clearance rate implies a high mutation rate, thus indicating that pharmacological mono-therapy will ultimately fail, since the virus can rapidly manifest a resistance to any one drug. More importantly, this knowledge directly contributed to the current practice of simultaneously administering multiple drugs to HIV positive individuals in an effort to counteract the high mutation rate of the virus.

Following its success in helping to identify this significant feature of the HIV pathogenesis, the use of mathematical modeling and parameter identification in the study of HIV experienced a dramatic increase. In particular, in the wake of the publication of [42], there were papers covering everything from additional and/or

H.T. Banks: Center for Research in Scientific Computation, North Carolina State University, Raleigh, Box 8205, NC 27695-8205, USA. e-mail: htbanks@eos . ncsu . edu

D.M. Bortz: Department of Mathematics, University of Michigan, 2074 East Hall, 525 East University Avenue, Ann Arbor, MI 48109-1109, USA. e-mail: dmbortz@umich . edu

Correspondence to: $\mathrm{H}$. T. Banks

Key words or phrases: Sensitivity Analysis - Parameter Sensitivity Methodology - HIV Models 
alternative compartment formulations $[13,27,33,37,43,48,55,59,60]$ to arguments for and against the use of delay differential equations in modeling the eclipse phase [19-21,30,32,34-36] (including those that addressed the solution stability [35, 36]). Moreover, in the context of delay equations, many of these papers focused heavily on the inter-relationship between the parameters describing the drug efficacy $\eta$, the length of the eclipse phase $\tau$, the infected T-cell death rate $\delta$, and the virion clearance rate $c[19,21,30,32,34-36]$. The purpose of this paper is to illustrate our approach, which allows one to develop new insights into HIV pathogenesis by utilizing a mathematical tool not typically associated with conventional NLS techniques. Indeed, there is a precedence for this approach, as is evidenced by previous papers within the HIV modeling literature that make use of stochastic analysis and inference $[24,51,52,57,61]$, control theory $[25,56]$, and nonlinear analysis [53]. Note that the above survey is not intended to be comprehensive, as there already exist thorough reviews of the field presented in [38,40,41].

For any system of differential equations designed to model real world phenomena, whether it be biological, chemical, or physical, a common goal is to understand the manner in which the system's constitutive parameters influence its solution. These parameters (such as $\delta$ above) are designed to correspond to aspects of the phenomena under investigation (e.g., HIV pathogenesis), and thus it is desirable to predict how changes in the parameters will affect the solution. Indeed, there are several papers in the HIV modeling field which focus heavily on the topic (good examples include $[48,50])$.

One way to address this question is to perform a sensitivity analysis, a mathematical tool developed in the context of modern control theory. Though not often isolated and identified, the sensitivity equations (used in sensitivity analyses) are actually quite pervasive in mechanical, aerospace, and electrical engineering [1, 16], as well as optimization and inverse problem theory. Furthermore, both the least squares and maximum likelihood (ML) based model discrimination/model selection literature employs sensitivity equations in calculating the the dispersion matrix and Fisher Information Matrix (FIM) respectively. In the least squares-based theory, the dispersion matrix is used to determine stability and accuracy functions [8]. In the ML-based theory, the inverse FIM is used in evaluating the Generalized Akaike Information Criteria (GAIC) [2] and the Information Complexity (ICOMP) [12] (see [11] for a survey of the field). In particular, utilization of AIC-type tests can be found in the ecology [39], biomedical engineering [15], and pharmaceutical sciences [47] literature.

A precursor to formal sensitivity analysis can be traced back to an 1833 electrostatics experiment designed to measure the inductance of certain metals [14]. However, significant activity in this area only arose in the middle part of the $20^{\text {th }}$ century, concomitant with the development of modern control theory in the late 1930's. In our analysis, we will employ the semirelative sensitivity function, though there are other possibilities, such as the logarithmic sensitivity function advocated by Bode in his book on electrical network analysis [7].

We direct the interested reader to the following introductory $[17,18,46]$ and advanced texts $[26,58]$ as well as Bortz and Nelson's sensitivity analysis of a delay differential equation model of in vivo HIV infection dynamics [10]. We also note 
that the sensitivity analysis described in this paper should not be confused with the statistical technique based on Latin Hypercube Sampling [6,23].

\subsection{Approach}

The first step in the sensitivity analysis is to derive the sensitivity equations by formally taking derivatives (with respect to a parameter of interest) on both sides of the original equation(s). The solution to this new system (assuming for the moment it is well-posed) contains information regarding the sensitivity of the original system to perturbations in the chosen parameter (around some a priori fixed value of that parameter). Hereafter we will refer to the solution to the sensitivity equations as a sensitivity function.

To illustrate the sensitivity procedure, we will examine an HIV population system with compartments described in [4], summarized in Table 1, and denoted by the vector $x=(V, A, C, T)^{T}$. In this case (see [4] for details), our system of distributed delay differential equations is

$$
\begin{aligned}
\dot{x}(t) & =L\left(x(t), x_{t}\right)+f_{1}(x(t))+f_{2}(t) \text { for } 0 \leq t \leq t_{f} \\
\left(x(0), x_{0}\right) & =(\Phi(0), \Phi) \in \mathbb{R}^{4} \times \mathscr{C}\left(-r, 0 ; \mathbb{R}^{4}\right),
\end{aligned}
$$

where $r$ and $t_{f}$ are finite, $x_{t}$ denotes the function $\theta \mapsto x(t+\theta), \theta \in[-r, 0]$, and for $(\eta, \phi) \in \mathbb{R}^{4} \times \mathscr{C}\left(-r, 0 ; \mathbb{R}^{4}\right)$,

$$
\begin{aligned}
L(\eta, \phi)= & {\left[\begin{array}{cccc}
-c & 0 & n_{C} & 0 \\
0 & r_{v}-\delta_{A} & 0 & 0 \\
0 & 0 & r_{v}-\delta_{C} & 0 \\
0 & 0 & 0 & r_{u}-\delta_{u}
\end{array}\right] \eta+n_{A}\left[\delta_{(1,2)}\right]_{(4,4)} \int_{-r}^{0} \phi(\theta) d P_{1}(\theta) } \\
& +\gamma\left(\left[\delta_{(3,2)}\right]_{(4,4)}-[\delta(2,2)]_{(4,4)}\right) \int_{-r}^{0} \phi(\theta) d P_{2}(\theta) \\
f_{1}(\eta)= & {\left[\begin{array}{c}
-p \eta_{1} \eta_{4} \\
-\delta\left(\sum_{i=2}^{4} \eta_{i}\right) \eta_{2}+p \eta_{1} \eta_{4} \\
-\delta\left(\sum_{i=2}^{4} \eta_{i}\right) \eta_{3} \\
-\delta\left(\sum_{i=2}^{4} \eta_{i}\right) \eta_{4}-p \eta_{1} \eta_{4}
\end{array}\right] } \\
f_{2}(t)= & {[0,0,0, S]^{T}, 0 \leq t \leq t_{f} . }
\end{aligned}
$$

Here the compartments in $x$ and the parameters (including the probability distributions $\left.P_{1}, P_{2}\right)$ given in the vector $q=\left(c, r_{v}, r_{u}, n_{A}, n_{C}, \delta, \delta_{A}, \delta_{C}, \delta_{u}, \gamma, p, P_{1}\right.$, $\left.P_{2}, S\right)^{T}$ are all described in [4]. A full and thorough sensitivity analysis could include not only derivatives with respect to the scalar parameters (e.g., $\gamma$ or $\delta_{A}$ ), but also Fréchet derivatives with respect to the delay distributions (e.g., $P_{1}$ or $P_{2}$ ). The following sections include discussions regarding the well-posedness of the sensitivity equations and an example numerical simulation as well as an interpretation of the results. 
Table 1. in vitro model compartments

\begin{tabular}{ll}
\hline Notation & Description \\
\hline$V$ & Infectious viral population \\
$A$ & Acutely infected cells \\
$C$ & Chronically infected cells \\
$T$ & Uninfected or target cells \\
\hline
\end{tabular}

\section{Theory}

For those interested in the mathematical considerations, this section contains theoretical foundations for the well-posedness of the sensitivity equations. While the results presented here are important because they legitimize our study of these equations, understanding the techniques in the proofs are not essential to appreciating the simulations and results presented in Section 3. Therefore, those readers who wish to skip the details in this section may do so with little loss in understanding the formal aspects of sensitivity analyses.

For our illustrative discussions, we will only consider distributions $P_{1}, P_{2}$ that are both differentiable and parameterizable by a mean $\mu$ and a standard deviation $\sigma$ (i.e., for $i=1,2, p_{i}(\theta)=\frac{\partial}{\partial \theta} P_{i}(\theta)$ and $P_{i}(\theta)=P_{i}\left(\theta ; \mu_{i}, \sigma_{i}\right)$ for $\left.\theta \in[-r, 0]\right)$. Moreover, we further assume that the resulting densities $p_{i}$ are $\mathscr{C}^{1}$ in $\mu_{i}$ and $\sigma_{i}$, respectively. To illustrate a sensitivity analysis, let us fix the forms of the distributions $P_{1}, P_{2}$ and consider for $t \in\left[-r, t_{f}\right]$, the derivative of $x\left(t ; \mu_{1}\right)$ with respect to $\mu_{1}$ (where $\mu_{1}$ is the parameter corresponding to the mean of $p_{1}$ ). If we let $(\eta, \phi) \in \mathbb{R}^{4} \times \mathscr{C}\left(-r, 0 ; \mathbb{R}^{4}\right), t \in\left[0, t_{f}\right], \mu_{1}>0$, then from results established in [9], we note that $\mathscr{F}\left(t, \eta, \phi, \mu_{1}\right)=L\left(\eta, \phi ; \mu_{1}\right)+f_{1}(\eta)+f_{2}(t)$ is $\mathscr{C}^{1}$ in $t, \eta, \phi$, and $\mu_{1}$ under smoothness assumptions (detailed in[4]) on $\mathscr{F}, L, f_{1}$, and $f_{2}$. For our specific case, to prove that the derivative of $x$ with respect to $\mu_{1}$ exists and is continuous in $t$, we will make use of the following lemma.

Lemma 1. There exists a solution to

$$
\begin{aligned}
\dot{y}(t) & =g_{1}\left(x\left(t ; \mu_{1}\right) ; y(t)\right)+g_{2}\left(\mu_{1} ; y_{t}\right)+g_{3}\left(x_{t}\left(\mu_{1}\right), \mu_{1} ; 1\right) \quad \text { for } 0 \leq t \leq t_{f} \\
\left(y(0), y_{0}\right) & =(\Psi(0), \Psi) \in \mathbb{R}^{4} \times \mathscr{C}\left(-r, 0 ; \mathbb{R}^{4}\right),
\end{aligned}
$$

for $x\left(t ; \mu_{1}\right)$ the solution to $(1)$, and where for $\mu, \xi \in \mathbb{R}, \eta, \zeta \in \mathbb{R}^{4}, \phi, \psi \in$ $\mathscr{C}\left(-r, 0 ; \mathbb{R}^{4}\right)$,

$$
\begin{aligned}
g_{1}(\eta ; \zeta)= & M_{\eta} \zeta \\
g_{2}(\mu ; \psi)= & n_{A}\left[\delta_{(1,2)}\right]_{(4,4)} \int_{-r}^{0} \psi(\theta) p_{1}\left(\theta ; \mu, \sigma_{1}\right) d \theta \\
& +\gamma\left(\left[\delta_{(3,2)}\right]_{(4,4)}-\left[\delta_{(2,2)}\right]_{(4,4)}\right) \int_{-r}^{0} \psi(\theta) p_{2}\left(\theta ; \mu_{2}, \sigma_{2}\right) d \theta \\
g_{3}(\phi, \mu ; \xi)= & n_{A}\left[\delta_{(1,2)}\right]_{(4,4)} \int_{-r}^{0} \phi(\theta)\left(\frac{\partial}{\partial \mu_{1}} p_{1}\left(\theta ; \mu, \sigma_{1}\right)\right)(\xi) d \theta,
\end{aligned}
$$


and where

$$
M_{\eta}=\left[\begin{array}{cccc}
-c-p \eta_{4} & 0 & n_{C} & -p \eta_{1} \\
p \eta_{4} & r_{v}-\delta_{A}-\delta\left(2 \eta_{2}+\eta_{3}+\eta_{4}\right) & -\delta \eta_{2} & -\delta \eta_{2}+p \eta_{1} \\
0 & -\delta \eta_{3} & r_{v}-\delta C-\delta\left(\eta_{2}+2 \eta_{3}+\eta_{4}\right) & -\delta \eta_{3} \\
-p \eta_{4} & -\delta \eta_{4} & -\delta \eta_{4} & r_{u}-\delta_{u}-\delta\left(\eta_{2}+\eta_{3}+2 \eta_{4}\right)-p \eta_{1}
\end{array}\right] .
$$

Proof. On the right side of (2), the function $g_{1}+g_{2}+g_{3}$ satisfies both the differentiability condition (Lemma 4.1) and the global Lipschitz condition (Lemma 4.2) from [4]. Following the reasoning in the proof of Theorem 4.5 in the same reference, by defining a convergent sequence of successive approximations, it can then easily be shown that a solution exists and is unique.

Remark 1. Note that Lemma 1 guarantees the existence of a solution to a system of equations with a general initial condition $\Psi$. Recall that in equation (1), the initial condition $\Phi$ is independent of $\mu_{1}$ and thus the next step will be to argue that system (2) combined with the trivial initial condition $\Psi=0$ comprises the sensitivity equations.

Theorem 1. For the solution $x$ of (1), $x$ has a derivative with respect to the parameter $\mu_{1}$ and for $\mu_{1}=\mu>0$, this derivative $v(t)=\frac{\partial}{\partial \mu_{1}} x(t ; \mu)$ satisfies (2) with the initial condition $(\Psi(0), \Psi)=(0,0) \in \mathbb{R}^{4} \times \mathscr{C}\left(-r, 0 ; \mathbb{R}^{4}\right)$.

Proof. To prove the existence of a derivative of $x$ with respect to the parameter $\mu_{1}$, we fix $\mu_{1}$ at $\mu>0$, let $\varepsilon \in \mathbb{R}$ be a perturbation of $\mu$, and for all $t \in\left[-r, t_{f}\right]$, define

$$
h(t, \mu, \varepsilon)=x(t ; \mu+\varepsilon)-x(t ; \mu) .
$$

The overall structure of the proof is thus to show that

$$
\frac{\partial}{\partial \mu_{1}} x(t ; \mu)=\lim _{|\varepsilon| \rightarrow 0} \frac{h(t, \mu, \varepsilon)}{\varepsilon}
$$

exists and is continuous for $t \in\left[-r, t_{f}\right]$. We begin by considering $h$

$$
\begin{aligned}
h(t, \mu, \varepsilon)= & \int_{0}^{t}\left\{\mathscr{F}\left(s, x(s ; \mu+\varepsilon), x_{s}(\mu+\varepsilon), \mu+\varepsilon\right)\right. \\
& \left.-\mathscr{F}\left(s, x(s ; \mu), x_{s}(\mu), \mu\right)\right\} d s \\
= & \int_{0}^{t}\left\{\mathscr{F}\left(s, x(s ; \mu+\varepsilon), x_{s}(\mu+\varepsilon), \mu+\varepsilon\right)\right. \\
& -\mathscr{F}\left(s, x(s ; \mu), x_{s}(\mu+\varepsilon), \mu+\varepsilon\right) \\
& +\mathscr{F}\left(s, x(s ; \mu), x_{s}(\mu+\varepsilon), \mu+\varepsilon\right)-\mathscr{F}\left(s, x(s ; \mu), x_{s}(\mu), \mu+\varepsilon\right) \\
& \left.+\mathscr{F}\left(s, x(s ; \mu), x_{s}(\mu), \mu+\varepsilon\right)-\mathscr{F}\left(s, x(s ; \mu), x_{s}(\mu), \mu\right)\right\} d s .
\end{aligned}
$$

According to the Mean Value Theorem [29], we have

$$
\begin{aligned}
h(t, \mu, \varepsilon)= & \int_{0}^{t} \int_{0}^{1}\left\{D_{x} \mathscr{F}\left(s, x(s ; \mu)+s^{\prime} h(s, \mu, \varepsilon), x_{s}(\mu+\varepsilon), \mu+\varepsilon\right)(h(s, \mu, \varepsilon))\right. \\
& +D_{x_{t}} \mathscr{F}\left(s, x(s ; \mu), x_{s}(\mu)+s^{\prime} h_{s}(\mu, \varepsilon), \mu+\varepsilon\right)\left(h_{s}(\mu, \varepsilon)\right) \\
& \left.+D_{\mu_{1}} \mathscr{F}\left(s, x(s ; \mu), x_{s}(\mu), \mu+s^{\prime} \varepsilon\right)(\varepsilon)\right\} d s^{\prime} d s,
\end{aligned}
$$


where $D_{x} \mathscr{F}, D_{x_{t}} \mathscr{F}, D_{\mu_{1}} \mathscr{F}$ are the Fréchet derivatives of $\mathscr{F}$ with respect to its second, third, and fourth arguments respectively. Since $\mathscr{F}$ is $\mathscr{C}^{1}$ in all its arguments, we then know that there exists linear functions $\Delta_{s^{\prime}, \varepsilon}^{1}, \Delta_{s^{\prime}, \varepsilon}^{2}, \Delta_{s^{\prime}, \varepsilon}^{3}$ (each parameterized by $s^{\prime}$ and $\varepsilon$ ) such that

$$
\begin{aligned}
h(t, \mu, \varepsilon)= & \int_{0}^{t} \int_{0}^{1}\left\{D_{x} \mathscr{F}\left(s, x(s ; \mu), x_{s}(\mu), \mu\right)(h(s, \mu, \varepsilon))+\Delta_{s^{\prime}, \varepsilon}^{1}(h(s, \mu, \varepsilon))\right. \\
& +D_{x_{t}} \mathscr{F}\left(s, x(s ; \mu), x_{s}(\mu), \mu\right)\left(h_{s}(\mu, \varepsilon)\right)+\Delta_{s^{\prime}, \varepsilon}^{2}\left(h_{s}(\mu, \varepsilon)\right) \\
& \left.+D_{\mu_{1}} \mathscr{F}\left(s, x(s ; \mu), x_{s}(\mu), \mu\right)(\varepsilon)+\Delta_{s^{\prime}, \varepsilon}^{3}(\varepsilon)\right\} d s^{\prime} d s
\end{aligned}
$$

where $\left|\Delta_{s^{\prime}, \varepsilon}^{1}\right|,\left|\Delta_{s^{\prime}, \varepsilon}^{2}\right|,\left|\Delta_{s^{\prime}, \varepsilon}^{3}\right| \rightarrow 0$ uniformly in $s^{\prime}$ as $|\varepsilon| \rightarrow 0$. Thus for $s \in[0, t]$, $\nu, \xi \in \mathbb{R}, \eta, \zeta \in \mathbb{R}^{4}, \phi, \psi \in \mathscr{C}\left(-r, 0 ; \mathbb{R}^{4}\right)$, and $g_{1}, g_{2}, g_{3}$ as defined in Lemma 1 ,

$$
\begin{aligned}
D_{x} \mathscr{F}(s, \eta, \phi, v)(\zeta) & =g_{1}(\eta ; \zeta) \\
D_{x_{t}} \mathscr{F}(s, \eta, \phi, \nu)(\psi) & =g_{2}(\nu ; \psi) \\
D_{\mu_{1}} \mathscr{F}(s, \eta, \phi, v)(\xi) & =g_{3}(\phi, v ; \xi) .
\end{aligned}
$$

Then the equation for $h$ is

$$
\begin{aligned}
h(t, \mu, \varepsilon)= & \int_{0}^{t}\left\{g_{1}(x(s ; \mu) ; h(s, \mu, \varepsilon))+g_{2}\left(\mu ; h_{s}(\mu, \varepsilon)\right)+g_{3}\left(x_{s}(\mu), \mu ; \varepsilon\right)\right\} d s \\
& +\int_{0}^{t} \int_{0}^{1}\left\{\Delta_{s^{\prime}, \varepsilon}^{1}(h(s, \mu, \varepsilon))+\Delta_{s^{\prime}, \varepsilon}^{2}\left(h_{s}(\mu, \varepsilon)\right)+\Delta_{s^{\prime}, \varepsilon}^{3}(\varepsilon)\right\} d s^{\prime} d s .
\end{aligned}
$$

Moreover, since $g_{1}, g_{2}, g_{3}$ are all linear in their last arguments, the equation for $h$ can be used to obtain

$$
\begin{aligned}
& \leq \max _{\tau \in[t-r, t]} \int_{0}^{\tau}\left\{\left|g_{1}(x(s ; \mu) ; \cdot)+\int_{0}^{1}\right| \Delta_{s^{\prime}, \varepsilon}^{1}\left|d s^{\prime}\right||h(s, \mu, \varepsilon)|\right. \\
& +\left|g_{2}(\mu ; \cdot)+\int_{0}^{1}\right| \Delta_{s^{\prime}, \varepsilon}^{2}\left|d s^{\prime}\right|\left\|h_{s}(\mu, \varepsilon)\right\| \\
& \left.+\left|g_{3}\left(x_{s}(\mu), \mu ; \cdot\right)+\int_{0}^{1}\right| \Delta_{s^{\prime}, \varepsilon}^{3}\left|d s^{\prime}\right||\varepsilon|\right\} d s,
\end{aligned}
$$

where $\|\cdot\|$ is the $\infty$-norm on $[t-r, t]$. Thus for constants $K_{1}, K_{2}>0$, we know that

$$
\left\|h_{t}(\mu, \varepsilon)\right\| \leq K_{1} \int_{0}^{t}\left\|h_{s}(\mu, \varepsilon)\right\| d s+K_{2} t_{f}|\varepsilon|,
$$

and a simple application of Gronwall's inequality implies that

$$
\left\|h_{t}(\mu, \varepsilon)\right\| \leq K_{2}\left|t_{f}\right||\varepsilon| \exp \left(K_{1} t_{f}\right),
$$

which will be useful in the next step. 
Now, if we divide both sides of the equation for $h$ by $\varepsilon$ so that

$$
\begin{aligned}
\frac{h(t, \mu, \varepsilon)}{\varepsilon}= & \int_{0}^{t}\left\{g_{1}\left(x(s, \mu) ; \frac{h(s, \mu, \varepsilon)}{\varepsilon}\right)+g_{2}\left(\mu ; \frac{h_{s}(\mu, \varepsilon)}{\varepsilon}\right)\right. \\
& \left.+g_{3}\left(x_{s}(\mu), \mu ; \frac{\varepsilon}{\varepsilon}\right)\right\} d s \\
& +\int_{0}^{t} \int_{0}^{1}\left\{\Delta_{s^{\prime}, \varepsilon}^{1}\left(\frac{h(s, \mu, \varepsilon)}{\varepsilon}\right)+\Delta_{s^{\prime}, \varepsilon}^{2}\left(\frac{h_{s}(\mu, \varepsilon)}{\varepsilon}\right)\right. \\
& \left.+\Delta_{s^{\prime}, \varepsilon}^{3}\left(\frac{\varepsilon}{\varepsilon}\right)\right\} d s^{\prime} d s,
\end{aligned}
$$

we note that the form of the integrand is strikingly similar to the right side of the equation in (2). For equation (2), we denote the solution generated using $\mu_{1}=\mu$ and initial condition $(\Psi(0), \Psi)=(0,0) \in \mathbb{R}^{4} \times \mathscr{C}\left(-r, 0 ; \mathbb{R}^{4}\right)$ as $v(t)$ for $t \in\left[-r, t_{f}\right]$. Moreover, we claim that this solution $v$ is equal to the limit of $h / \varepsilon$ as $|\varepsilon| \rightarrow 0$.

By Lemma 1, we know that $v$ exists and is continuous for $t \in\left[-r, t_{f}\right]$. Clearly $v$ and $h / \varepsilon$ are identically zero for $t \in[-r, 0]$ for all $\varepsilon>0$, and thus we consider for $t \in\left[0, t_{f}\right]$

$$
\begin{aligned}
\mid v(t) & -\frac{h(t, \mu, \varepsilon)}{\varepsilon} \mid \leq\left\|v_{t}-\frac{h_{t}(\mu, \varepsilon)}{\varepsilon}\right\|_{\infty} \\
\leq & \max _{\tau \in[t-r, t]} \mid \int_{0}^{\tau}\left\{g_{1}\left(x(s, \mu) ; v(s)-\frac{h(s, \mu, \varepsilon)}{\varepsilon}\right)+g_{2}\left(\mu ; v_{s}-\frac{h_{s}(\mu, \varepsilon)}{\varepsilon}\right)\right. \\
& \left.+g_{3}\left(x_{s}(\mu), \mu ; 1-\frac{\varepsilon}{\varepsilon}\right)\right\} d s-\int_{0}^{\tau} \int_{0}^{1}\left\{\Delta_{s^{\prime}, \varepsilon}^{1}\left(\frac{h(s, \mu, \varepsilon)}{\varepsilon}\right)\right. \\
& \left.+\Delta_{s^{\prime}, \varepsilon}^{2}\left(\frac{h_{s}(\mu, \varepsilon)}{\varepsilon}\right)+\Delta_{s^{\prime}, \varepsilon}^{3}\left(\frac{\varepsilon}{\varepsilon}\right)\right\} d \tau d s \mid \\
\leq & \max _{\tau \in[t-r, t]}\left\{\int _ { 0 } ^ { \tau } \left\{\left|g_{1}(x(s, \mu) ; \cdot)\right|\left|v(s)-\frac{h(s, \mu, \varepsilon)}{\varepsilon}\right|\right.\right. \\
& +\left|g_{2}(\mu ; \cdot)\right|\left|v_{s}-\frac{h_{s}(\mu, \varepsilon)}{\varepsilon} \|+\right| g_{3}\left(x_{s}(\mu), \mu ; \cdot\right)|| 0 \mid \\
& \left.\left.+\int_{0}^{1}\left\{\left|\Delta_{s^{\prime}, \varepsilon}^{1}\right|\left|\frac{h(s, \mu, \varepsilon)}{\varepsilon}\right|+\left|\Delta_{s^{\prime}, \varepsilon \mid}^{2}\right|\left|\frac{h_{s}(\mu, \varepsilon)}{\varepsilon}\|\|_{\infty}+\right| \Delta_{s^{\prime}, \varepsilon}^{3}|| 1 \mid\right\} d s^{\prime}\right\} d s\right\} \\
\leq & \int_{0}^{t}\left(\left|g_{1}(x(s, \mu) ; \cdot)\right|+\left|g_{2}(\mu ; \cdot)\right|\right) \mid v_{s}-\frac{h_{s}(\mu, \varepsilon)}{\varepsilon} \|_{\infty} d s \\
& +\int_{0}^{t} \int_{0}^{1}\left\{\left|\Delta_{s^{\prime}, \varepsilon}^{1}\right|\left|\frac{h(s, \mu, \varepsilon)}{\varepsilon}\right|+\left|\Delta_{s^{\prime}, \varepsilon}^{2}\right|\left|\frac{h_{s}(\mu, \varepsilon)}{\varepsilon}\|\|_{\infty}+\right| \Delta_{s^{\prime}, \varepsilon}^{3}|| 1 \mid\right\} d s^{\prime} d s .
\end{aligned}
$$

By equation (3), we know that

$$
\begin{aligned}
\| v_{t} & -\frac{h_{t}(\mu, \varepsilon)}{\varepsilon}\left\|_{\infty} \leq \int_{0}^{t}\left\{\left|g_{1}(x(s, \mu) ; \cdot)\right|+\left|g_{2}(\mu ; \cdot)\right|\right\} v_{s}-\frac{h_{s}(\mu, \varepsilon)}{\varepsilon}\right\|_{\infty} d s \\
& +\int_{0}^{t} \int_{0}^{1} K_{2}\left|t_{f}\right| \exp \left(K_{1} t_{f}\right)\left\{\left|\Delta_{s^{\prime}, \varepsilon}^{1}\right|+\left|\Delta_{s^{\prime}, \varepsilon}^{2}\right|\right\} \frac{|\varepsilon|}{|\varepsilon|}+\left|\Delta_{s^{\prime}, \varepsilon}^{3}\right||1| d s^{\prime} d s
\end{aligned}
$$




$$
\begin{aligned}
\leq & K_{1} \int_{0}^{t}\left\|v_{s}-\frac{h_{s}(\mu, \varepsilon)}{\varepsilon}\right\|_{\infty} d s \\
& +t_{f} \int_{0}^{1}\left\{K_{2} t_{f} \exp \left(K_{1} t_{f}\right)\left\{\left|\Delta_{s^{\prime}, \varepsilon}^{1}\right|+\left|\Delta_{s^{\prime}, \varepsilon}^{2}\right|\right\}+\left|\Delta_{s^{\prime}, \varepsilon}^{3}\right|\right\} d s^{\prime} \\
\leq & K_{1} \int_{0}^{t}\left\|v_{s}-\frac{h_{s}(\mu, \varepsilon)}{\varepsilon}\right\|_{\infty} d s+K_{3}\left(t_{f}\right) \int_{0}^{t}\left\{\left|\Delta_{s^{\prime}, \varepsilon}^{1}\right|+\left|\Delta_{s^{\prime}, \varepsilon}^{2}\right|+\left|\Delta_{s^{\prime}, \varepsilon}^{3}\right|\right\} d s^{\prime},
\end{aligned}
$$

where $K_{3}\left(t_{f}\right)=t_{f} \max \left\{K_{2} t_{f} \exp \left(K_{1} t_{f}\right), 1\right\}$. By Gronwall's inequality, we then have that

$$
\left|v(t)-\frac{h(t, \mu, \varepsilon)}{\varepsilon}\right| \leq K_{3}\left(t_{f}\right) \int_{0}^{1}\left\{\left|\Delta_{s^{\prime}, \varepsilon}^{1}\right|+\left|\Delta_{s^{\prime}, \varepsilon}^{2}\right|+\left|\Delta_{s^{\prime}, \varepsilon}^{3}\right|\right\} d s^{\prime} e^{K_{1} t_{f}} .
$$

Since $\left|\Delta_{s^{\prime}, \varepsilon}^{1}\right|,\left|\Delta_{s^{\prime}, \varepsilon}^{2}\right|,\left|\Delta_{s^{\prime}, \varepsilon}^{3}\right| \rightarrow 0$ uniformly in $s^{\prime}$ as $|\varepsilon| \rightarrow 0$, we can then conclude that for $t \in\left[-r, t_{f}\right], h / \varepsilon \rightarrow v$ as $|\varepsilon| \rightarrow 0$. Therefore, the partial derivative of $x$ with respect to $\mu_{1}$ (evaluated at $\mu_{1}=\mu>0$ ) exists and satisfies (2) with the initial condition $(\Psi(0), \Psi)=(0,0) \in \mathbb{R}^{4} \times \mathscr{C}\left(-r, 0 ; \mathbb{R}^{4}\right)$, which completes the proof.

Remark 2. The line of reasoning presented here in Lemma 1 and Theorem 1 concerns the existence and continuity (in $t$ ) of the derivative of a solution to (1) with respect to the specific parameter $\mu_{1}$. Similar arguments (with minimal changes to $g_{3}$ ) establish the existence and continuity (in $t$ ) of derivatives with respect to $\mu_{2}$, $\sigma_{1}$, and $\sigma_{2}$. For the parameters that appear in (1) as linear coefficients, $g_{1}$ and $g_{2}$ are slightly altered (dependent upon the parameter under consideration), while $g_{3} \equiv 0$. However, these differences do not change the conclusion that the derivative of the solution $x(t)$ (with respect to any parameter appearing on the right side of (1)) exists and is continuous in time. One can also establish differentiability of solutions with respect to discrete delays (i.e., when $P_{1}$ or $P_{2}$ is a Dirac measure) and well-posedness of the appropriate sensitivity equations. The arguments, while in the spirit of those given above, are however somewhat more tedious and will not be given here.

\section{Analysis and results}

In this section we examine some applications of the theory developed in Section 2. All of the simulations presented in this section were done using Matlab software originally developed in [4] for the purpose of simulating systems of Abstract Evolution Equations (AEE's) that are linear in the delay (e.g., system (1)). As can be inferred from equation (2), in order to solve sensitivity equations, one needs the solution $x$ of the original system. Therefore, we use the calculated solution from [4] with parameters $q^{*} \in Q_{a d}$ (the space of admissible parameters) identified by minimizing the cost

$$
J(q)=\sum_{i=1}^{10}\left(X\left(t_{i} ; q\right)-\widehat{X}_{i}\right)^{2},
$$

over $q \in Q_{a d}$, where $\widehat{X}=\left\{\widehat{X}_{i}\right\}$ is the data from [45] taken at times $\left\{t_{i}\right\}$, and $X=A+C+T$. In the calculations reported on here and in [4,9], we employed the Nelder-Mead nonlinear iterative Matlab optimization routine fminsearch to 
estimate the 10 parameters consisting of those in $q$ in Section 1.2 minus $c, r_{v}, r_{u}$, and $S$ which were held fixed and given (see [4] for more details).

We are not able, however, to compute the exact solution $x$ and thus (as described in $[4,9])$ we actually minimize

$$
J^{N}(q)=\sum_{i=1}^{10}\left(X^{N}\left(t_{i} ; q\right)-\widehat{X}_{i}\right)^{2},
$$

where $x^{N}=\left(V^{N}, A^{N}, C^{N}, T^{N}\right)$ is an appropriate approximation to $x, N$ is an integer describing the accuracy of the numerical simulation such that $\lim _{N \rightarrow \infty} x^{N}(t ; q)$ $=x(t ; q)$, and $X^{N}=A^{N}+C^{N}+T^{N}$. In this case, we used the piecewise linear spline approximation scheme for delay differential equations as developed by Banks and Kappel in [5]. For our example, these approximation schemes lead to a $4(N+1)$ dimensional nonlinear ordinary differential equation vector system for the generalized Fourier coefficients in the expansion of solutions in terms of $N+1$ piecewise linear spline basis elements. We employed $N=32$ in our calculations to obtain 33 basis elements and a $4 \times 33=132$ dimensional approximating system. The numerical scheme (also described in [4,9]) is such that as $N \rightarrow \infty$, a minimizer $q^{N *}$ to (5) converges to $q^{*}$, a minimizer to (4). Both the experimental results and the numerical best fit solution $x^{N}$ (using parameters $q^{N *}$ from Table 2) are depicted in Figure 1.

By Theorem 1, we can legitimately consider the derivative of both sides of (1) with respect to any appropriate parameter. We first consider the derivative of $x(t)$ with respect to $\mu_{1}$ at $\mu_{1}=\mu$

$$
\begin{aligned}
\frac{d}{d \mu_{1}} \dot{x}(t ; \mu)= & \frac{d}{d \mu_{1}} L\left(x(t ; \mu), x_{t}(\mu) ; \mu\right)+\frac{d}{d \mu_{1}} f_{1}(x(t ; \mu)) \\
& +\frac{d}{d \mu_{1}} f_{2}(t) \text { for } 0 \leq t \leq t_{f} \\
\frac{d}{d \mu_{1}}\left(x(0, \mu), x_{0}(\mu)\right)= & \frac{d}{d \mu_{1}}(\Phi(0), \Phi) \in \mathbb{R}^{4} \times \mathscr{C}\left(-r, 0 ; \mathbb{R}^{4}\right) .
\end{aligned}
$$

Table 2. Optimal in vitro model parameter values.

\begin{tabular}{lll}
\hline Parameter & Value & Units \\
\hline$n_{A}$ & 0.112 & hours $^{-1}$ \\
$n_{C}$ & 0.011 & hours $^{-1}$ \\
$\gamma$ & $9 E-4$ & hours $^{-1}$ \\
$\delta_{A}$ & 0.078 & hours $^{-1}$ \\
$\delta_{C}$ & 0.025 & hours $^{-1}$ \\
$\delta_{u}$ & 0.017 & hours $^{-1}$ \\
$\delta$ & $1 E-12$ & (cell hours $^{-1}$ \\
$p$ & $1.3 E-6$ & (cell hours) $^{-1}$ \\
$\mu_{1}$ & -22.8 & hours \\
$\mu_{2}$ & -26 & hours \\
\hline
\end{tabular}




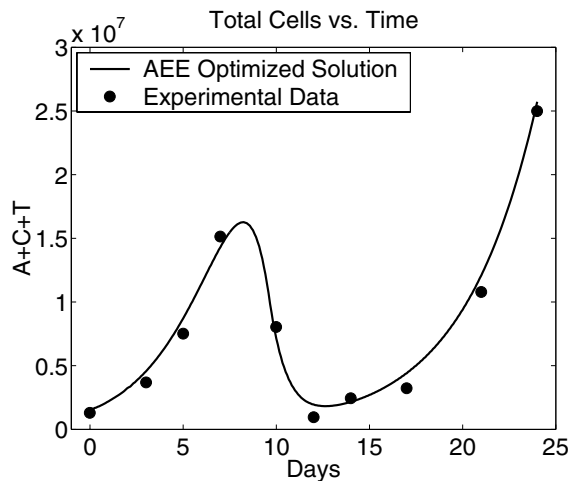

Fig. 1. Data from [45] and best fit simulation $x^{N}$ of (1).

If we denote $v(t)=\frac{d}{d \mu_{1}} x(t ; \mu)$ (for some specific value of $\mu_{1}=\mu>0$ ), we obtain the sensitivity equations

$$
\begin{aligned}
\dot{v}(t) & =g_{1}(x(t ; \mu) ; v(t))+g_{2}\left(\mu ; v_{t}\right)+g_{3}\left(x_{t}(\mu), \mu ; 1\right) \text { for } 0 \leq t \leq t_{f} \\
\left(v(0), v_{0}\right) & =(0,0) \in \mathbb{R}^{4} \times \mathscr{C}\left(-r, 0 ; \mathbb{R}^{4}\right),
\end{aligned}
$$

where $g_{1}, g_{2}, g_{3}$ are as defined in Lemma 1. As before, due to the complexity of the right side of (7), we cannot solve exactly for the solution $v(t)$. Moreover, we do not have $x$ which appears in the terms $g_{1}$ and $g_{3}$; we only have an approximation $x^{N}$ to $x$. Therefore, we must propose a viable numerical scheme to calculate an approximation $v^{N}$ to solutions of (7), with $x$ replaced by $x^{N}$ such that $\lim _{N \rightarrow \infty} v^{N}=v$.

Hence we consider $v^{N}$ an approximate solution to (7) with $x=x^{N}$ in the coefficients. This is a linear nonautonomous system of the form

$$
\begin{aligned}
\dot{v}^{N}(t) & =\mathscr{A}^{N}(t) v^{N}(t)+g_{2}\left(v_{t}^{N}\right)+g_{3}\left(x_{t}^{N}\right) \text { for } 0 \leq t \leq t_{f} \\
\left(v^{N}(0), v_{0}^{N}\right) & =(0,0) \in \mathbb{R}^{4} \times \mathscr{C}\left(-r, 0 ; \mathbb{R}^{4}\right),
\end{aligned}
$$

where $N$ is fixed, $x^{N}$ is given, and $\mathscr{A}^{N}$ maps $\mathbb{R}^{4} \times \mathscr{C}\left(-r, 0 ; \mathbb{R}^{4}\right)$ to $\mathbb{R}^{4}$. Note that this is a special case of the systems treated in [4], where existence and uniqueness are guaranteed. To obtain convergence of $v^{N}$ to $v$ (the unique solution to (7)), we turn to [3]. A straightforward extension of the theory presented in [3] to treat nonautonomous linear systems such as (8) will yield, (under the approximation scheme described in [4]), the desired convergence.

If we were to plot simulations of (7) (or actually, the approximate solutions defined by (8)), interpretations of these plots would suggest specific effects that changes in $\mu_{1}$ would have on the solution $x$. Moreover, if we were to also perform the analogous derivation for the infection rate $p$, a plot of that sensitivity function would depict the effect that changes in $p$ would have on $x$. Since $\mu_{1}$ and $p$ differ in their units, the sensitivity functions for $\mu_{1}$ and $p$ would also have different units, thus rendering any comparison meaningless. We turn to the sensitivity analysis literature to resolve this issue. To enable a comparison of the effects that parameters 
with different units have on the solution, we simply multiply by the parameter under consideration, e.g., $\left(\frac{\partial}{\partial \mu_{1}} x_{1}(t ; \mu), \frac{\partial}{\partial \mu_{1}} x_{2}(t ; \mu), \frac{\partial}{\partial \mu_{1}} x_{3}(t ; \mu), \frac{\partial}{\partial \mu_{1}} x_{4}(t ; \mu)\right) \cdot \mu$. This form of the sensitivity function is known as the semirelative or semilogarithmic or unnormalized sensitivity function [17,18]. Moreover, this form is actually the differential of $x$ with respect to $\mu_{1}$ at $\mu$ in the direction $\mu$

$$
D_{\mu_{1}} x_{i}(t ; \mu)[\mu]=\left(\frac{\partial}{\partial \mu_{1}} x_{i}(t ; \mu)\right) \cdot \mu
$$

for $i=1,2,3,4$. With this weighting, we now have the tools to rank the parameters with regard to their influence over the solution.

Figure 2 depicts the approximation $v^{N}$ of the solution $v$ to (7) (at $\mu=-22.8$ ), with each compartment multiplied by $\mu$. It is important to realize that while the $y$-axis in Figure 2 has units of cells or virions respectively, it should still be thought of as a plot reflecting changes in the state with respect to changes in $\mu_{1}$. In other words, we interpret the upper-left plot of Figure 2 to suggest that for a (positive) change in the mean delay, the virion compartment $V$ will be dramatically smaller just before day 10 and then larger around day 12 (relative to $V(t ;-22.8)$ ). Likewise for a change in $\mu_{1}$, the acutely infected cell compartment $A$ will be slightly smaller around day 9 and dramatically larger around day 10 (relative to $A(t ;-22.8)$ ). All
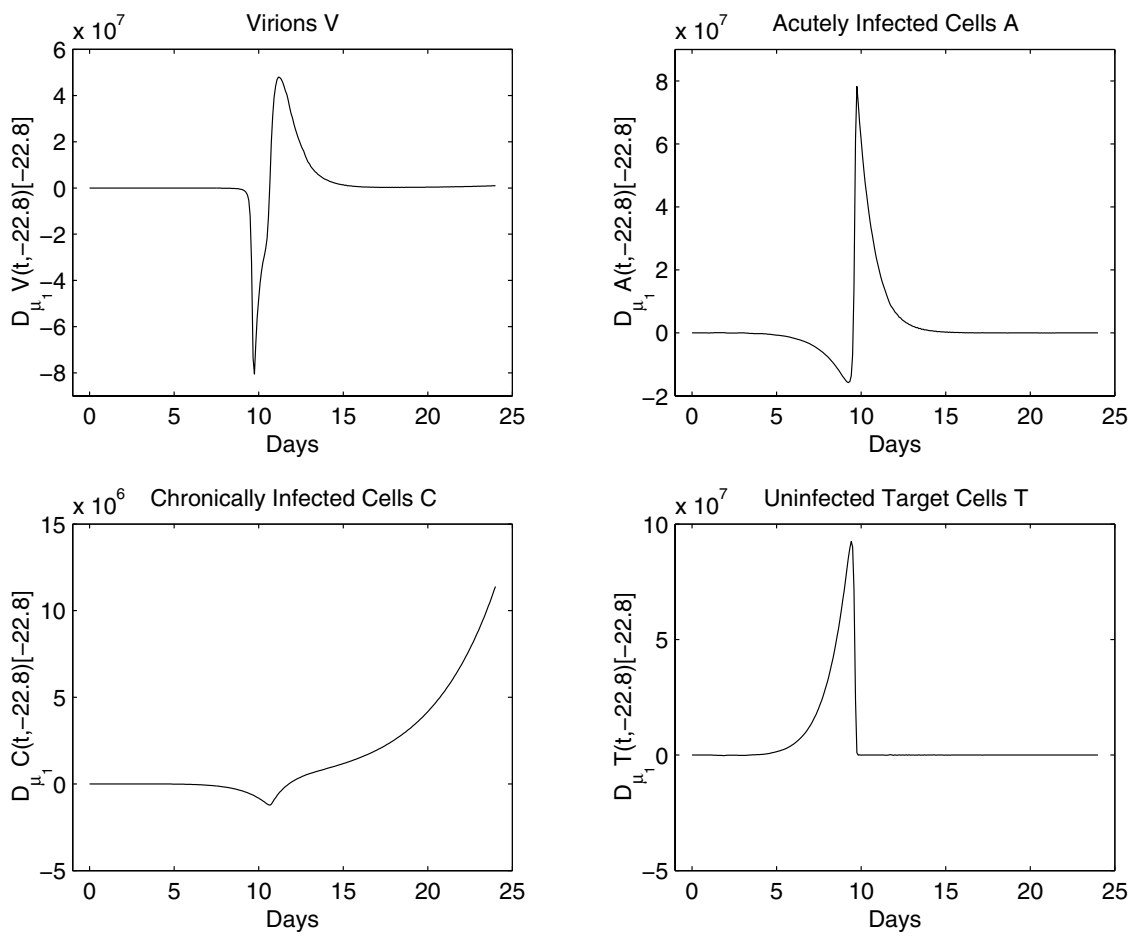

Fig. 2. Simulation of the semirelative sensitivity solution with respect to $\mu_{1}$ at $\mu_{1}=\mu=$ -22.8 . 
the plots depicted in Figure 2 suggest that there will be dramatic changes in the solution for changes in $\mu_{1}$, and indeed Figure 3 supports this claim (as well as the specific predictions suggested by the interpretation of Figure 2). For this simulation, it is important to note that there is practically no indication that the solution $x$ will exhibit any sensitivity to $\mu_{1}$ until around day 5. In other words, for simulations on a short time interval (i.e., $t \in[-r, 120]$ hours), one could easily conclude that the solution $x$ is insensitive to $\mu_{1}$ (in the neighborhood of $\mu_{1}=\mu=-22.8$ hours).

As another example, let us consider the solution parameterized with respect to the infection rate $p$, i.e., $x(t)=x(t ; p)$. Thus the derivative of $(1)$ with respect to $p$ at $\tilde{p}=1.3 \times 10^{-6}$ is

$$
\begin{aligned}
\frac{d}{d p} \dot{x}(t ; \tilde{p})= & \frac{d}{d p} L\left(x(t ; \tilde{p}), x_{t}(\tilde{p})\right)+\frac{d}{d p} f_{1}(x(t ; \tilde{p}), \tilde{p}) \\
& +\frac{d}{d p} f_{2}(t) \text { for } 0 \leq t \leq t_{f} \\
\frac{d}{d p}\left(x(0, \tilde{p}), x_{0}(\tilde{p})\right)= & \frac{d}{d p}(\Phi(0), \Phi) \in \mathbb{R}^{4} \times \mathscr{C}\left(-r, 0 ; \mathbb{R}^{4}\right) .
\end{aligned}
$$

As mentioned in the last part of Section 2, the sensitivity equations with respect to different parameters will be slightly different than (7), but unique solutions still
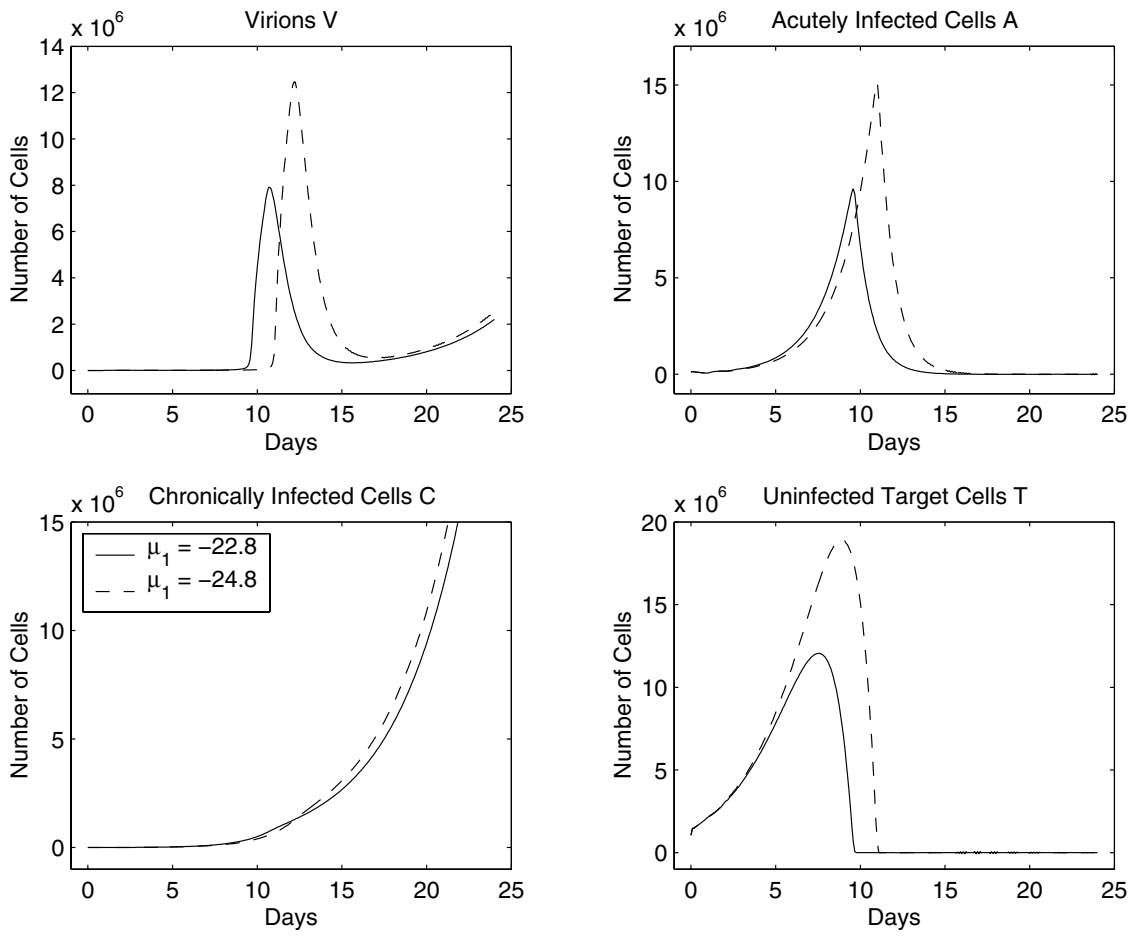

Fig. 3. Simulations of $x^{N}(t ;-24.8)$ and $x^{N}(t ;-22.8)$. 
exist and are continuous (for each system of sensitivity equations). Figures 4 and 5 depict the semirelative sensitivity functions for $p$ and $\mu_{2}$, respectively. A comparison of the scales on the vertical axis in Figure 2 versus the axis in Figures 4 and 5 suggests that changes in $\mu_{1}$ have a more significant influence in the solution $x$ than changes in $\mu_{2}$ or $p$ (and in one of the compartments by over four orders of magnitude). This result coincides nicely with one of the primary conclusions from [4], in which we concluded that when fitting the data, adding the second delay between than acute and chronic infection was not as significant as inclusion of the delay between viral infection and viral production.

Now that we have established the framework for calculating semirelative sensitivity functions, let us consider how to rank the influence that changes in the individual parameters have upon the solution $x$. Clearly, there are many options, but for simplicity, we will rank the parameters according to the magnitude of the $\infty$-norm, e.g., for the virion compartment and the sensitivity with respect to $\delta_{A}$, we consider

$$
\max _{t \in\left[0, t_{f}\right]}\left|D_{\delta_{A}} V(t ; 0.0776)[0.0776]\right| .
$$

To illustrate our reasoning, we will focus on just the virion compartment $V$. Of the parameters over which we performed our nonlinear least squares estimation in [4],
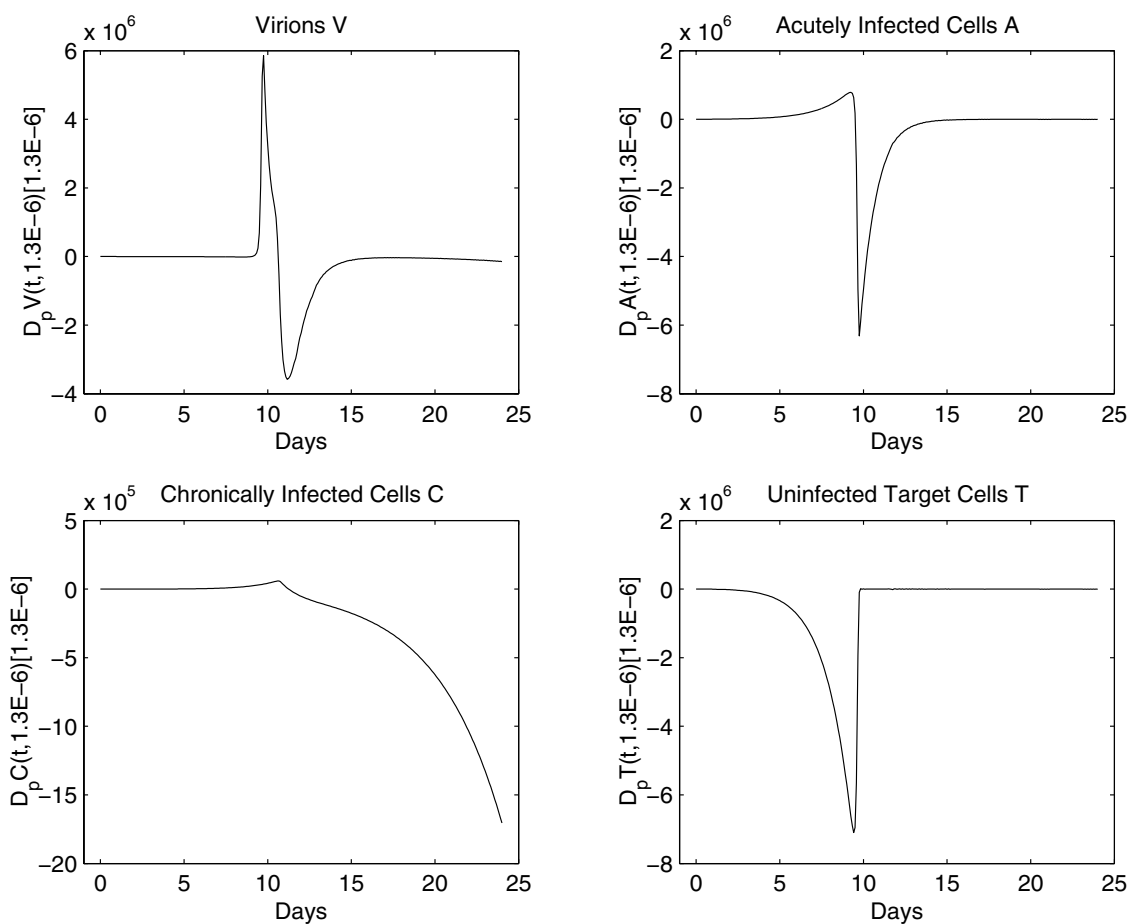

Fig. 4. Simulation of semirelative sensitivity solution with respect to the infection rate $p$ for $\tilde{p}=1.3 \times 10^{6}$. 

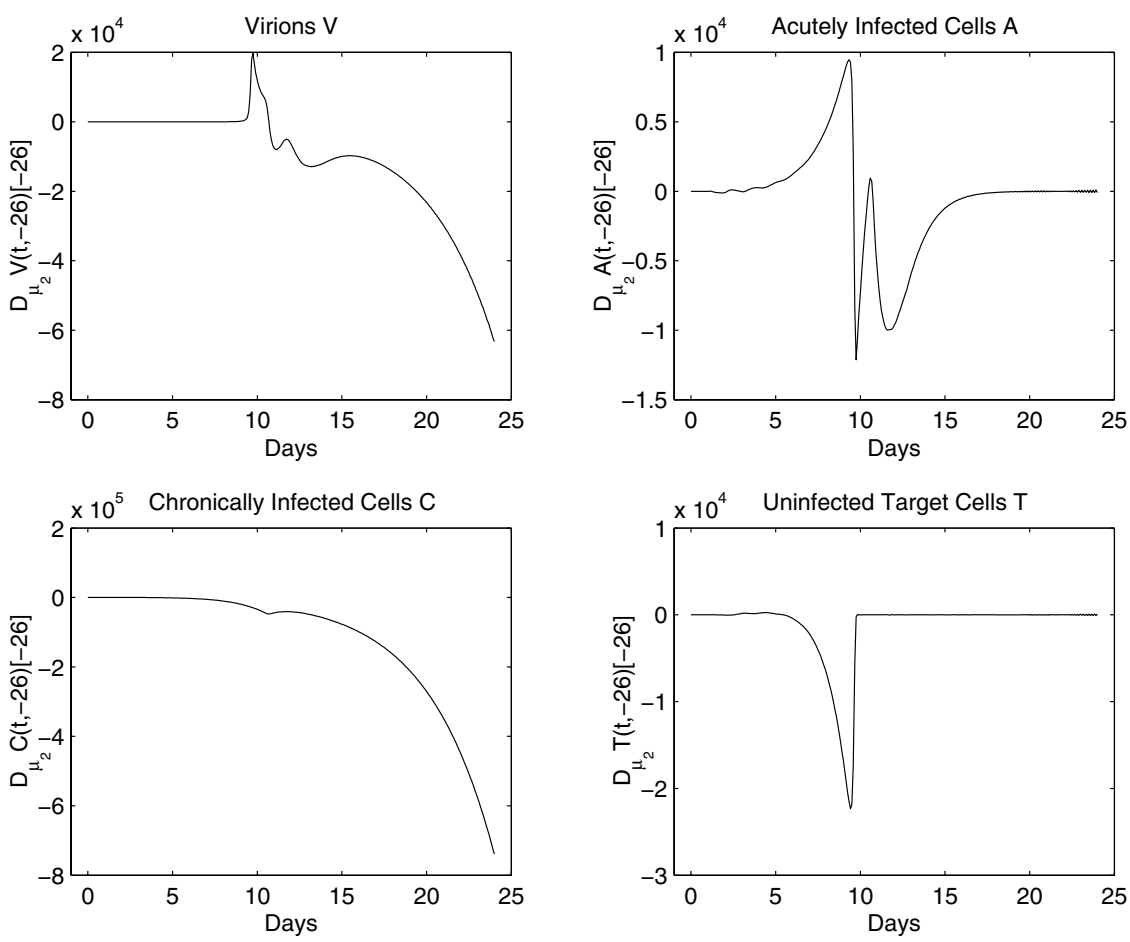

Fig. 5. Simulation of semirelative sensitivity solution with respect to the mean delay between acute and chronic infection $\mu_{2}$ for $\tilde{\mu}_{2}=-26$.

the chosen metric was largest for the parameters $\mu_{1}, n_{A}, \delta_{A}$, and $\delta_{u}$. Figure 6 depicts (for the compartment $V$ ), the absolute values of the semirelative sensitivity functions with respect to $\mu_{1}, n_{A}, \delta_{A}$, and $\delta_{u}$, for $t \in[8.5,15]$ (the domain where there

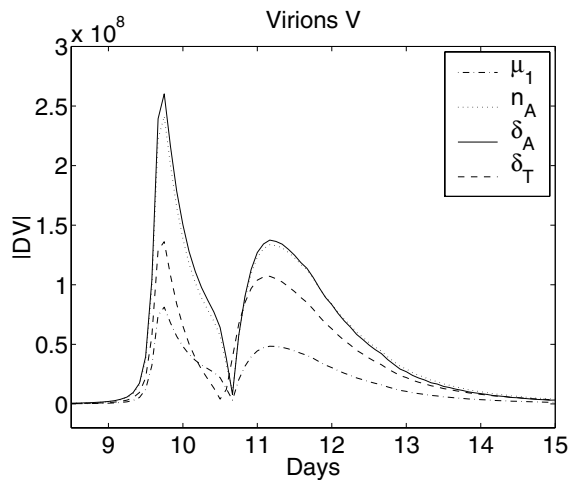

Fig. 6. Absolute value of simulations of semirelative sensitivity solutions for several parameters ( $V$ compartment only). 
is the most activity in the sensitivity functions). The interpretation of this figure strongly suggests that $\delta_{A}$ and $n_{A}$ have the strongest influence over the solution in the virion compartment (in the chosen $\infty$-norm). Therefore, for the use of equation (1) (as a model to simulate HIV pathogenesis), both the viral production rate and the death rate for acutely infected cells ( $n_{A}$ and $\delta_{A}$ respectively) should be given top priority when choosing which parameters to determine with a high degree of accuracy. In other words, these parameters play an important role in the model and obtaining good values for them is more important to the system response than good values for other parameters to which solutions are less sensitive.

\section{Concluding remarks}

As discussed in Section 1.1, the taking of a derivative (with respect to parameters) of the equations governing a system is not a new idea and indeed has been around (in some form) for at least 170 years. Within control theory and engineering applied to physical systems, the forms of the fundamental mathematical models often are, for the most part, relatively well established and not so open to debate. For example, in some investigations, it may not be fruitful to question the significance of the viscosity parameter in the Navier-Stokes equations (although sensitivity of flow patterns to viscosity is sometimes very important, see [49]). However, the constitutive parameters and forms of the mathematical models employed in the biological sciences are frequently not as well agreed upon, and indeed (as is evidenced by the literature) open to considerable debate. Since the current approach to sensitivity was originally developed in the context of control theory, the cited literature is (understandably) biased toward that field; a considerable proportion of the papers are devoted to analyzing the sensitivity of transfer functions and eigenvalues. Thus the application of mathematically rigorous sensitivity analyses to dynamical systems designed to model biological phenomena does not seem to be common practice. Indeed, many sensitivity studies often involve copious simulations. As such, there are many possibilities that have not been fully examined.

In the analysis presented in this paper, we only considered first derivatives of the state variables. In theory, we could have formally differentiated (1) with respect to multiple parameters (e.g., $\frac{\partial^{2} x}{\partial n_{A} \partial \delta_{A}}$ or $\frac{\partial^{2} x}{\partial c^{2}}$ ), an analysis of which could be used to ascertain the independent identifiability of parameters. Furthermore, consideration of the Hessian matrix of parameter sensitivities would yield local curvature and confidence interval information (we did not do this for the example discussed in this paper since a careful statistical analysis is perhaps not so useful given the paucity of observations (10 points) in the one data set used in our inverse problem calculations in [4]). We could have also taken a derivative with respect to the initial conditions, which (as is intuitive) would suggest the influence of the initial conditions over the solution (this can be extremely useful in certain biological investigations). Finally, we could have considered the derivative of the least squares functional (4) with respect to a parameter (as was explored in [28]), which could then be used as part of a Jacobian in an optimization algorithm (as part of a parameter estimation scheme). 
The process of taking the derivative of a system with respect to a parameter is typically not an exceedingly challenging task. Moreover, it is important to remember that the sensitivity function only reveals the local behavior since it is, in essence, a Taylor Series expanded around a fixed set of parameter values. However, this idea can yield useful insights into the solution of complex systems (even those with nonlinearities and delays) such as (1). Effectively, the technique of using simulation sensitivity functions presented in this paper is a more efficient (and mathematically rigorous) way to attain insight into a system than manually adjusting a parameter and observing the effect on the solution through massive simulation efforts.

Acknowledgements. This research was supported in part by the Air Force Office of Scientific Research under grants AFOSR-F49620-01-1-0026 and AFOSR-F49620-98-1-0430 and in part through a GAANN Fellowship to D. M. Bortz under Department of Education grant P200A980801. We also wish to thank Drs. Sarah Holte and Michael Emerman for the use of their experimental data as well as stimulating discussions during the course of this research.

\section{References}

1. Adelman, H.M., Haftka, R.T.: Sensitivity analysis of discrete structural systems. A.I.A.A. J. 24, 823-832 (1986)

2. Akaike, H.: A new look at the statistical model identification. IEEE Transactions on Automatic Control 19, 716-723 (1974)

3. Banks, H.T.: Identification of nonlinear delay systems using spline methods. In: V. Lakshmikantham, (ed.), Nonlinear Phenomena in Mathematical Sciences, Academic Press, Inc., New York, NY, 1982, pp. 47-55

4. Banks, H.T., Bortz, D.M., Holte, S.E.: Incorporation of variability into the mathematical modeling of viral delays in HIV infection dynamics. Mathematical Biosciences 183, 63-91 (2003)

5. Banks, H.T., Kappel, F.: Spline approximations for functional differential equations. J. Differential Equations 34, 496-522 (1979)

6. Blower, S.M., Dowlatabadi, H.: Sensitivity and uncertainty analysis of complex models of disease transmission: an HIV model, as an example. International Statistics Review 62, 229-243 (1994)

7. Bode, H.W.: Network Analysis and Feedback Amplifier Design. Van Nostrand New York, NY, 1945

8. Borowiak, D.S.: Model Discrimination for Nonlinear Regression Models. Vol. 101, of Stastics: textbooks and monographs. Marcel Dekker, Inc. New York, NY, 1989

9. Bortz, D.M.: Modeling, Analysis, and Estimation of an in vitro HIV Infection Using Functional Differential Equations. Ph.D. dissertation North Carolina State University, Raleigh, NC, 2002

10. Bortz, D.M., Nelson, P.W.: Sensitivity analysis of nonlinear lumped parameter models of HIV infection dynamics. Bulletin of Mathematical Biology 66 (2004), pp. 1009-1026.

11. Bozdogan, H.: Akaike's information criterion and recent developments in information compelexity. J. Math. Psychology 44, 62-91 (2000)

12. Bozdogan, H., Haughton, D.M.A.: Informational complexity criteria for regression models. Comput. Stat. Data Anal. 28, 51-76 (1998)

13. Callaway, D.S., Perelson, A.S.: HIV-1 Infection and low steady state viral loads. Bulletin of Math. Biol. 64, 29-64 (2002) 
14. Christie, S.H.: The Bakerian Lecture: Experimental determination of the laws of magneto-electric induction in different masses of the same metal and of its intensity in different metals. Philosophical Transactions of the Royal Society of London 123, 95$142(1833)$

15. Christini, D.J., Bennett, F.M., Lutchen, K.R., Ahmed, H.M., Hausdorff, J.M., Oriol, N.: Application of linear and nonlinear time-series modeling to heart-rate dynamics analysis. IEEE Transactions on Biomedical Engineering 42(4), 411-415 (1995)

16. Cruz, J.B.: System Sensitivity Analysis. Dowden Hutchinson \& Ross, Inc., Stroudsburg, PA, 1973

17. Eslami, M.: Theory of Sensitivity in Dynamic Systems: An Introduction. Springer-Verlag, Berlin, 1994

18. Frank, P.M.: Introduction to System Sensitivity Theory. Academic Press, Inc., New York, NY, 1978

19. Grossman, Z., Feinberg, M., Kuznetsov, V., Dimitrov, D., Paul, W.: HIV infection: how effective is drug combination treatment?. Immunology Today 19, 528-532 (1998)

20. Grossman, Z., Polis, M., Feinberg, M.B., Grossman, Z., Levi, I., Jankelevich, S., Yarchoan, R., Boon, J., de Wolf, F., Lange, J.M.A., Goudsmit, J., Dimitrov, D.S., Paul, W.E.: Ongoing HIV dissemination during HAART Nature Medicine 5, 1099-1104 (1999)

21. Herz, A.V.M., Bonhoeffer, S., Anderson, R.M., May, R.M., Nowak, M.A.: Viral dynamics in vivo: limitations on estimates of intracellular delay and virus decay. Proceedings of the National Academy of Sciences, USA 93, 7247-7251 1996

22. Ho, D.D., Neumann, A.U., Perelson, A.S., Chen, W., Leonard, J.M., Markowitz, M.: Rapid turnover of plasma virions and CD4 lymphocytes in HIV-1 infection Nature 373, 123-126 (1995)

23. R. L. Iman and J. C. Helton An investigation of uncertainty and sensitivity analysis techniques for computer models. Risk Analysis 8, 71-90 (1988)

24. Kamina, A., Makuch, R.W., Zhao, H.: Stochastic modeling of early HIV-1 population dynamics. Mathematical Biosciences 170, 187-198 (2001)

25. D. Kirschner and S. Lenhart and S. Serbin Optimal control of chemotherapy of HIV. J. Math. Biol. 35, 775-792 (1997)

26. Kleiber, M., Antúnez, H., Hien, T.D., Kowalczyk, P.: Parameter Sensitivity in Nonlinear Mechanics: Theory and Finite Element Computations. John Wiley \& Sons New York, NY, 1997

27. Kramer, I.: Modeling the dynamical impact of HIV on the immune system: Viral clearance, infection, and AIDS Mathematical and Computer Modelling 29, 95-112 (1999)

28. Kubiak, S., Lehr, H., Levy, R., Moeller, T., Parker, A., Swim, E.: Modeling control of HIV infection through structured treatment interruptions with recommendations for experimental protocol. In: Proceedings of the 2001 Industrial Mathematics Modeling Workshop for Graduate Students, no. CRSC-TR01-27 in Center for Research in Scientific Computation Techical Report, North. Carolina. State University, Raleigh, NC, Nov. 2001

29. Lang, S.: Analysis II, 1969 Addison-Welsey Publishing Company. Inc., Reading, MA, 1969

30. Lloyd, A.L.: The dependence of viral parameter estimates on the asumed viral load life cycle: limitations of studies of viral load data, Proceedings of the Royal Society of London Series B 268, 847-854 (2001)

31. Mittler, J.E., Markowitz, M., Ho, D.D., Perelson, A.S.: Improved estimates for HIV-1 clearance rate and intracellular delay. AIDS 13, 1415-1417 (1999)

32. Mittler, J.E., Sulzer, B., Neumann, A.U., Perelson, A.S.: Influence of delayed viral production on viral dynamics in HIV-1 infected patients. Mathematical Biosciences 152, 143-163 (1998) 
33. Murray, J.M., Kaufmann, G., Kelleher, A.D., Cooper, D.A.: A model of primary HIV-1 infection. Mathematical Biosciences 154, 57-85 (1998)

34. Nelson, P.W., Mittler, J.E., Perelson, A.S.: Effect of drug efficacy and the eclipse phase of the viral life cycle on estimates of HIV viral dynamic parameters. J. Acquired Immune Deficiency Syndromes 26, 405-412 (2001)

35. Nelson, P.W., Murray, J.D., Perelson, A.S.: A model of HIV-1 pathogenesis that includes an intracellular delay. Mathematical Biosciences 163, 201-215 (2000)

36. Nelson, P.W., Perelson, A.S.: Mathematical analysis of delay differential equation models of HIV-1 infection. Mathematical Biosciences 179, 73-94 (2002)

37. Nowak, M.A., Bonhoeffer, S., Shaw, G.M., May, R.M.: Anti-viral drug treatment: dynamics of resistance in free virus and infected cell populations. J. Theoretical Biology 184, 203-217 (1997)

38. Nowak, M.A., May, R.M.: Virus Dynamics: Mathematical Principles of Immunology and Virology. Oxford University Press, Inc., New York, NY, 2000

39. Pease, C.M., Mattson, D.J.: Demography of the yellowstone grizzly bears. Ecology 80 957-975 (1999)

40. Perelson, A.S.: Modeling viral and immune system dynamics. Nature Reviews Immunology 2, 28-36 (2002)

41. Perelson, A.S., Nelson, P.W.: Mathematical analysis of HIV-1 dynamics in vivo. SIAM Review 41, 3-44 (1999)

42. Perelson, A.S., Neumann, A.U., Markowitz, M., Leonard, J.M., Ho, D.D.: HIV-1 dynamics in vivo: virion clearance rate infected cell life-span and viral generation time Science. 271, 1582-1586 (1996)

43. Phillips, A.N.: Reduction of HIV concentration during acute infection: Independence from a specific immune response Science. 271, 497-499 (1996)

44. Ramratnam, B., Bonhoeffer, S., Binley, J., Hurley, A., Zhang, L., Mittler, J.E., Markowitz, M., Moore, J.P., Perelson, A.S., Ho, D.D.: Rapid production and clearance of HIV-1 and hepatitis C virus assessed by large volume plasma apheresis. The Lancet 354, 1782-1785 (1999)

45. Rogel, M.E., Wu, L.I., Emerman, M.: The human immunodeficiency virus type $1 \mathrm{vpr}$ gene prevents cell proliferation during chronic infection. J. Virology 69, 882-888 (1995)

46. Saltelli, A., Chan, K., Scott, E.M. eds.: Sensitivity Analysis, Wiley Series in Probability and Statistics. John Wiley \& Sons New York, NY, 2000

47. Smith, B.P., Brier, M.E.: Statistical approach to neural network model building for gentamicin peak predictions. J. Pharmaceutical Sciences 85, 65-69 (1996)

48. Stafford, M.A., Corey, L., Cao, Y., Daar, E.S., Ho, D.D., Perelson, A.S.: Modeling plasma virus concentration during primary HIV infection. J. Theoretical Biology 203, 285-301 (2000)

49. Stanley, L.G.: Computational Methods for Sensitivity Analysis with Applications for Elliptic Boundary Value Problems. Ph.D. dissertation Virginia Polytechnic Institute and State University Blacksburg, VA, 1999

50. Stilianakis, N.I., Dietz, K., Schenzle, D.: Analysis of a model for the pathogenesis of AIDS Mathematical Biosciences. 145, 27-46 (1997)

51. Tan, W., Wu, H.: Stochastic modeling of the dynamics of CD4+ T-cell infection by HIV and some monte carlo studies. Mathematical Biosciences 147, 173-205 (1998)

52. Tuckwell, H.C., Le Corfec, E.: A Stochastic model for early HIV-1 population dynamics. J. Theoretical Biology 195, 451-463 (1998)

53. Verotta, D., Schaedeli, F.: Non-linear dynamics models characterizing long-term virological data from AIDS clinical trials. Mathematical Biosciences 176, 163-183 (2002) 
54. Wei, X., Ghosh, S.K., Taylor, M.E., Johnson, V.A., Emini, E.A., Deutsch, P., Lifson, J.D., Bonhoeffer, S., Nowak, M.A., Hahn, B.H., Saag, M.S., Shaw, G.M.: Viral dynamics in human immunodeficiency virus type 1 infection. Nature 373, 117-122 (1995)

55. Wein, L.M., D'Amato, R.M., Perelson, A.S.: Mathematical analysis of antiretroviral therapy aimed at HIV-1 eradication or maintenance of low viral loads. J. Theoretical Biology 192, 81-98 (1998)

56. Wein, L.M., Zeinos, S.A., Nowak, M.A.: Dynamic multidrug therapies for HIV: A control theoretic approach. J. Theoretical Biology 185, 15-29 (1997)

57. Wick, D., Self, S.G.: Early HIV Infection in vivo: Branching-process model for studying timing of immune responses and drug therapy. Mathematical Biosciences 165, 115-134 (2000)

58. Wierzbicki, A.: Models and Sensitivity of Control Systems, no. 5 in Studies in Automation and Control. Elsevier Science Publishing Company. Inc., New York, NY, 1984

59. Wodarz, D., Jansen, V.A.A.: The role of T cell help for anti-viral CTL responses. J. Theoretical Biology 211, 419-432 (2001)

60. Wodarz, D., Lloyd, A.L., Jansen, V.A.A., Nowak, M.A.: Dynamics of macrophage and $\mathrm{t}$ cell infection by HIV. J. Theoretical Biology 196, 101-113 (1999)

61. Wu, H., Ding, A.A., de Gruttola, V.: Estimation of HIV dynamic parameters. Statistics in Medicine 17, 2463-2485 (1998) 